\title{
Nilpotency and periodic points in non-uniform cellular automata
}

\author{
Supreeti Kamilya ${ }^{1} \cdot$ Jarkko Kari $^{2}$ (D
}

Received: 29 January 2020 / Accepted: 26 September 2020

(c) The Author(s) 2020

\begin{abstract}
Nilpotent cellular automata have the simplest possible dynamics: all initial configurations lead in bounded time into the unique fixed point of the system. We investigate nilpotency in the setup of one-dimensional non-uniform cellular automata (NUCA) where different cells may use different local rules. There are infinitely many cells in NUCA but only a finite number of different local rules. Changing the distribution of the local rules in the system may drastically change the dynamics. We prove that if the available local rules are such that every periodic distribution of the rules leads to nilpotent behavior then so do also all eventually periodic distributions. However, in some cases there may be non-periodic distributions that are not nilpotent even if all periodic distributions are nilpotent. We demonstrate such a possibility using aperiodic Wang tile sets. We also investigate temporally periodic points in NUCA. In contrast to classical uniform cellular automata, there are NUCA - even reversible equicontinuous ones-that do not have any temporally periodic points. We prove the undecidability of this property: there is no algorithm to determine if a NUCA with a given finite distribution of local rules has a periodic point.
\end{abstract}

\section{Introduction}

A one-dimensional cellular automaton (CA) consists of an infinite line of cells that evolve in discrete time following some local update rule. The CA is classical or uniform (UCA) if all the cells have the same local rule. Otherwise the CA is known as a hybrid or a nonuniform CA (NUCA). In this work all NUCA are assumed to have finitely many different local rules. A distribution is a bi-infinite sequence that identifies the rule used by each cell. The distribution is assumed to remain unchanged over time. It is not surprising that same

Research supported by the Academy of Finland grant 296018 and by the Government of India SPARC Grant P189.

Jarkko Kari

jkari@utu.fi

Supreeti Kamilya

kamilyasupreeti779@gmail.com

1 Indian Institute of Engineering Science and Technology, Shibpur, Howrah, India

2 Department of Mathematics and Statistics, University of Turku, 20014 Turku, Finland 
local rules may induce wildly different dynamics under different distributions, and it is then of interest to analyze the sets of rule distributions that yield a desired property [6-8]. For example, it is known that for any finite set of local rules the distributions that yield a surjective (resp. an injective) NUCA form a sofic shift (resp. a $\zeta$-rational set) [7].

Nilpotency is a property studied previously mostly for uniform cellular automata. In a nilpotent uniform CA, all initial configurations eventually lead to a particular uniform fixed point configuration. If this happens, the fixed point is reached from all configurations uniformly within bounded time. Equivalently, every individual cell eventually enters the same quiescent state, regardless of the initial configuration [11]. There is no algorithm to determine if a given one-dimensional uniform cellular automaton is nilpotent $[1,13]$, and this fact has turned out to be a fruitful source of reductions to prove further undecidability results concerning the eventual behavior of one-dimensional uniform cellular automata. For example, it implies that equicontinuity is an undecidable property [10], and that all nontrivial properties concerning the limit set of one-dimensional uniform cellular automata are undecidable [14].

In this paper we consider the property of nilpotency for non-uniform CA. We show that if all periodic rule distributions are nilpotent, so are all eventually periodic rule distributions. However, it is possible that some non-periodic distribution yields a non-nilpotent NUCA even if all periodic distributions are nilpotent.

We also consider temporally periodic points, i.e., points that have highly regular time evolution. In chaotic systems one has such regularity everywhere in the phase space interweaved with more complex transitive behavior. In one of the most widely used definitions of chaos—due to Devaney [9]—one indeed requires a chaotic dynamical system to be topologically transitive and sensitive to initial conditions, but also to have temporally periodic points densely in the phase space. An outstanding open problem for uniform cellular automata, due to F. Blanchard and P. Tisseur (see Section 25 in [3]), asks whether every surjective cellular automaton has a dense set of temporally periodic configurations, which would then imply that every transitive cellular automaton is Devaney chaotic. For reversible uniform cellular automata the property is known to hold since spatially periodic configurations are dense in the configuration space and they are automatically temporally periodic. However, in the non-uniform setting the situation is very different: we show that there are reversible equicontinuous NUCAs without any temporally periodic configurations. We also prove that it is undecidable to establish whether a given NUCA has any temporally periodic configurations. All considered rule distributions are very simple.

The paper is organized as follows. We first give basic definitions and terminologies in Sect. 2 . We then consider in Sect. 3 temporally periodic points in non-uniform CA and prove that it is undecidable if there are any. In Sect. 4 we turn to nilpotency of non-uniform cellular automata and prove that there are local rules such that every periodic arrangement of the rules yields a nilpotent NUCA while some non-periodic arrangement makes the NUCA non-nilpotent.

\section{Definitions and terminologies}

\subsection{Cellular automata}

Let $A$ be a finite state set. A one-dimensional configuration over $A$ is any function $c: \mathbb{Z} \longrightarrow A$ that assigns a state to every cell $i \in \mathbb{Z}$. We usually use the subscript notation and denote by $c_{i}$ 
the state of cell $i$ in configuration $c \in A^{\mathbb{Z}}$. A finite pattern over $A$ is an assignment $p \in A^{D}$ of states on a finite domain $D \subseteq \mathbb{Z}$ of cells.

A local rule is a function $f: A^{n} \longrightarrow A$ where $n \in \mathbb{Z}_{+}$is the width of the neighborhood. A synchronous application of the local rule $f$ using offset $a \in \mathbb{Z}$ at all cells defines a uniform cellular automaton $F: A^{\mathbb{Z}} \longrightarrow A^{\mathbb{Z}}$ by

$$
\left(\forall c \in A^{\mathbb{Z}}\right)(\forall i \in \mathbb{Z}) F(c)_{i}=f\left(c_{i+a}, c_{i+a+1}, \ldots, c_{i+a+n-1}\right) .
$$

For $r \in \mathbb{Z}_{+}$, a radius- $r$ cellular automaton has a local rule of width $2 r+1$ and offset $-r$. We also use radius $-\frac{1}{2}$ cellular automata that have width 2 local rules and offset 0 , i.e., the new state of each cell only depends on the old states of the cell and its immediate right neighbor. Uniform one-dimensional radius-1 CA with state set $A=\{0,1\}$ are known as elementary cellular automata (ECA), and we refer to these by their Wolfram number [20]. Note that every cellular automaton is a radius- $r$ cellular automaton for any large enough $r$.

Let $R$ be a finite set of radius- $r$ local rules $A^{2 r+1} \rightarrow A$. A non-uniform cellular automaton (NUCA) is specified by a distribution $b \in R^{\mathbb{Z}}$ where $b_{i}$ identifies the local rule used at cell $i$. The NUCA specified by distribution $b$ is hence the transformation $F_{b}: A^{\mathbb{Z}} \longrightarrow A^{\mathbb{Z}}$ where

$$
\left(\forall c \in A^{\mathbb{Z}}\right)(\forall i \in \mathbb{Z}) F_{b}(c)_{i}=b_{i}\left(c_{i-r}, c_{i-r+1}, \ldots, c_{i+r}\right) .
$$

Note that in this work the term "distribution" has no probabilistic flavour. It always refers to the spatial sequence of local rules in a NUCA.

We call NUCA $F_{b}$ injective (surjective, bijective) if every configuration has at most one pre-image (at least one pre-image, exactly one pre-image, respectively) under $F_{b}$. We call $F_{b}$ reversible if it is bijective and the inverse function $F_{b}^{-1}$ is also NUCA. There are bijective NUCAs that are not reversible since the inverse function may require infinitely many different local rules. (See our Corollary 1 and the discussion below it.)

For any finite set $S$, let $\sigma_{S}: S^{\mathbb{Z}} \longrightarrow S^{\mathbb{Z}}$ be the left shift map defined by $\sigma_{S}(x)_{i}=x_{i+1}$, for all $x \in S^{\mathbb{Z}}$ and $i \in \mathbb{Z}$. We may also denote $\sigma_{S}$ by $\sigma$ when $S$ is clear from the context. We call two sequences $x, y \in S^{\mathbb{Z}}$ asymptotic if $x_{i}=y_{i}$ for all but finitely many $i \in \mathbb{Z}$. We use this terminology both for distributions $(S=R)$ and configurations $(S=A)$. We also call $x \in S^{\mathbb{Z}}$

- uniform if $\sigma_{S}(x)=x$, and more precisely a-uniform for $a \in S$ if $x_{i}=a$ for all $i \in \mathbb{Z}$,

- finite, or more precisely $a$-finite for $a \in S$, if $x_{i}=a$ for all but finitely many $i \in \mathbb{Z}$,

- eventually constant if $x=\ldots a$ a $u b b \ldots$ for some $a, b \in S$ and $u \in S^{*}$,

- (spatially) periodic if $\sigma_{S}^{k}(x)=x$ for some $k \geq 1$, that is, if $x=\ldots p p p \ldots$ for a finite word $p \in S^{+}$,

- (spatially) eventually periodic if $x=\ldots p$ p uq $q \ldots$ for some $p, q \in S^{+}$and $u \in S^{*}$.

Note that a uniform CA is just a NUCA $F_{b}$ defined by a uniform distribution $b$. Note also the following simple implications:

$$
\begin{aligned}
& \text { uniform } \Rightarrow \text { finite } \Rightarrow \text { eventually constant } \Rightarrow \text { eventually periodic, } \\
& \text { uniform } \Rightarrow \text { periodic } \Rightarrow \text { eventually periodic. }
\end{aligned}
$$

In [6], three types of NUCAs are defined: $d v$-CA, $p v$-CA and $r v$-CA. Among these, $r v$-CA are NUCAs as defined above, $p v$-CA are NUCA with an eventually periodic distribution and $d \nu$-CA are NUCAs with a finite distribution of rules.

Let $F: A^{\mathbb{Z}} \longrightarrow A^{\mathbb{Z}}$ be a NUCA. A configuration $c \in A^{\mathbb{Z}}$ is temporally periodic if $F^{p}(c)=c$ for some $p \geq 1$ called the period, and it is a fixed point if $F(c)=c$. A configuration $c \in A^{\mathbb{Z}}$ is an eventually temporally periodic point if $F^{n}(c)$ is temporally periodic for some natural $n$ which is possibly different from zero. While in uniform CA 
Fig. 1 A space-time diagram of the uniform xor ECA 102 and a trace at cell 0

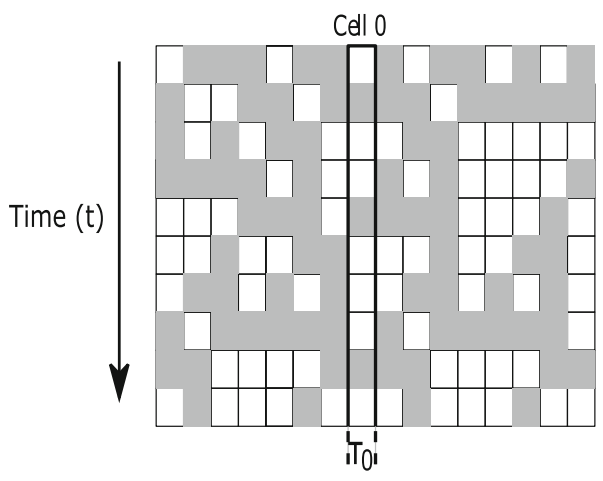

every spatially periodic configuration is automatically eventually temporally periodic, the same is no longer true for non-uniform CA.

A space-time diagram of a NUCA $F$ is a pictorial representation of an orbit, that is, an infinite sequence of configurations $\left(c_{t}\right)_{t \in \mathbb{N}}$ where $c_{t+1}=F\left(c_{t}\right)$. Fig. 1 shows a sample space-time diagram that is represented by a successive sequence of configurations, from top to bottom.

For a given configuration $c \in A^{\mathbb{Z}}$, the trace $\tau_{i} \in A^{\mathbb{N}}$ of cell $i$ in the orbit of $c$ is the $i$ 'th column in the space-time diagram of $c$, that is, the sequence defined as

$$
(\forall j \in \mathbb{N}) \tau_{i}(j)=F^{j}(c)_{i} .
$$

Figure 1 shows also the trace of cell 0.

Nilpotent cellular automata have trivial dynamics: NUCA $F: A^{\mathbb{Z}} \longrightarrow A^{\mathbb{Z}}$ is nilpotent if $F^{n}\left(A^{\mathbb{Z}}\right)$ is a singleton set for some $n \geq 1$. Clearly the unique element of $F^{n}\left(A^{\mathbb{Z}}\right)$ then must be a unique fixed point of $F$. More generally, NUCA $F$ is equicontinuous if for every cell $i \in \mathbb{Z}$ there is a finite set $D \subseteq \mathbb{Z}$ of cells such that for any configuration $c$ the finite pattern $c_{\mid D}$ uniquely determines the trace $\tau_{i}$ :

$$
(\forall i \in \mathbb{Z})(\exists \text { finite } D \subseteq \mathbb{Z})\left(\forall c, e \in A^{\mathbb{Z}}\right) c_{\mid D}=e_{\mid D} \Longrightarrow(\forall n \geq 0) F^{n}(c)_{i}=F^{n}(e)_{i} .
$$

This is equivalent to the formulation that $\left\{F, F^{2}, F^{3}, \ldots\right\}$ is an equicontinuous set of functions under the metric

$$
(c, e) \mapsto 2^{-\min \left\{|i| \mid c_{i} \neq e_{i}\right\}} \quad \text { if } c \neq e
$$

on $A^{\mathbb{Z}}$. (See [16], for example, or the few first pages of [5] for details on the standard prodiscrete topology on $A^{\mathbb{Z}}$ that this metric induces. Note that $A^{\mathbb{Z}}$ is a compact metric space so the notions of uniform equicontinuity and pointwise equicontinuity coincide.) Clearly every nilpotent NUCA is equicontinuous since traces may depend on the initial configuration only until the NUCA reaches its fixed point. A uniform CA is known to be equicontinuous if and only if it is eventually temporally periodic, that is, $F^{n+p}=F^{n}$ for some $n$ and $p \geq 1$ [15] but such characterization is no longer valid for non-uniform $C A$. In fact, there are equicontinuous NUCAs without any temporally periodic points (see Corollary 1 in Sect. 3).

\subsection{Wang tiles and cellular automata}

Let $\mathrm{C}$ be a finite set of colors. A Wang tile is an element of $C^{4}$, and a Wang tile set $T$ is a finite set of Wang tiles. Tile $(N, E, S, W)$ is conveniently drawn as a unit square on the 
Fig. 2 A NW-deterministic tile set $T$ contains at most one matching $c \in T$ for every $a, b \in T$

Fig. 3 NW-deterministic Wang tile set

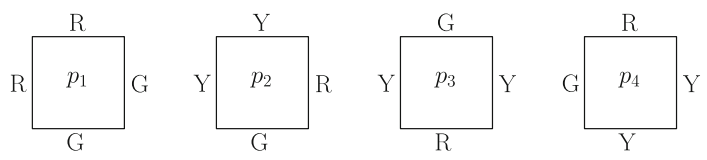

Fig. 4 Four consecutive diagonals tiles using the tiles of Fig. 3

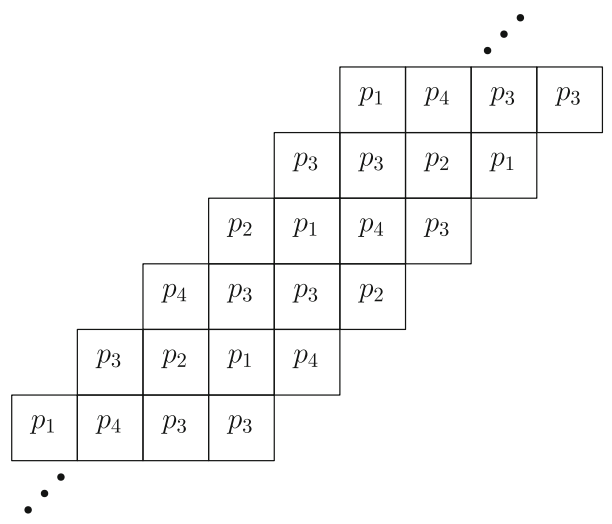

plane whose north, east, south and west edges have colors $N, E, S$ and $W$, respectively. A two-dimensional configuration $t \in T^{\mathbb{Z}^{2}}$, that is, an assignment $t: \mathbb{Z}^{2} \longrightarrow T$ of tiles on the infinite grid, is a tiling admitted by $T$ if, in every position $(i, j) \in \mathbb{Z}^{2}$, the north and west colors of tile $t(i, j)$ are the same as the south and east colors of tiles $t(i, j+1)$ and $t(i-1, j)$, respectively. In other words, the abutting edges of neighboring tiles must have the same color. A fundamental result by R.Berger states that it is undecidable if a given Wang tile set admits a tiling [2].

A two-dimensional configuration $t \in T^{\mathbb{Z}^{2}}$ is periodic if there exists $(n, m) \in \mathbb{Z}^{2} \backslash\{(0,0)\}$ such that $t(i, j)=t(i+n, j+m)$ for all $(i, j) \in \mathbb{Z}^{2}$, and we then call $(n, m)$ a period of $t$. If $t$ has some vertical and horizontal periods $(n, 0)$ and $(0, m)$ then we call $t$ two-periodic. If a Wang tile set admits a periodic tiling then it also admits a two-periodic tiling [19]. A Wang tile set is called aperiodic if it admits some tiling but it does not admit any periodic tilings. Such aperiodic tile sets exist [2], the smallest one contains 11 tiles [12].

A Wang tile set $T$ is called $N W$-deterministic if $(N, E, S, W),\left(N, E^{\prime}, S^{\prime}, W\right) \in T$ implies that $E=E^{\prime}$ and $S=S^{\prime}$, for all $E, E^{\prime}, S, S^{\prime} \in C$, that is, the north and the west colors identify each tile uniquely. See Fig. 2 for an illustration. For any infinite diagonal $d$ of tiles in the North-East to South-West orientation on the plane there is then at most one assignment of tiles on the diagonal below that matches in colors with $d$. See Fig. 3 for an example of a NW-deterministic tile set and Fig. 4 for a sample of four consecutive diagonals.

This naturally leads to the idea of considering the diagonals of tilings as configurations of a one-dimensional CA that maps a diagonal to the unique matching diagonal below. As in [13] we thus associate to a NW-deterministic tile set a one-dimensional, radius- $\frac{1}{2}$ uniform cellular automaton $F_{T}$ with state set $S=T \cup\{q\}$, where $q \notin T$ is a new symbol, and with 
local rule $f_{T}: S^{2} \longrightarrow S$ where

$$
f_{T}(a, b)=\left\{\begin{aligned}
c, & \text { if } a, b \in T \text { and } \exists c \in T \text { such that the } \\
& \text { colors between } a, b, c \text { match in Fig. } 2 \\
q, & \text { otherwise. }
\end{aligned}\right.
$$

It is easy to see that

- $F_{T}$ is nilpotent if and only if $T$ does not admit a tiling, and

- $F_{T}$ has more than one temporally periodic point if and only if $T$ admits a periodic tiling. (Note that the $q$-uniform configuration is automatically a fixed point and hence temporally periodic.)

As it is undecidable whether a given NW-deterministic tile set admits a tiling [13], or whether it admits a periodic tiling [17], we immediately obtain that it is undecidable whether a given one-dimensional uniform cellular automaton is nilpotent, or whether it has more than one temporally periodic point.

\section{Temporal periodicity in non-uniform CAs}

In uniform CA, all spatially periodic configurations eventually enter a temporally periodic cycle, so UCA always have at least one temporally periodic point. The following proposition shows that this is no longer true for non-uniform CA. Figure 5 illustrates a sample space-time diagram from the initial configuration of all $0 \mathrm{~s}$.

Proposition 1 Consider a binary state NUCA $F$ whose cell 0 uses the local rule of ECA 51 (the cell alternates between states 0 and 1 regardless of its neighbors) or the local rule of ECA 255 (state becomes 1 in every neighborhood pattern), and cells $i<0$ use the local rule of the ECA 102 (the next state of each cell is the modulo 2 sum on itself and its right neighbor). Then $F$ does not have any temporally periodic configurations.

Proof Assume, to the contrary, that $c \in\{0,1\}^{\mathbb{Z}}$ is temporally periodic for $F$, so there is $p>0$ such that $F^{p}(c)=c$. Let $\tau_{i} \in\{0,1\}^{\mathbb{N}}$ be the trace of cell $i$ in the orbit of $c$, that is,

$$
(\forall j \in \mathbb{N}) \tau_{i}(j)=F^{j}(c)_{i} .
$$

Each $\tau_{i}$ is $p$-periodic. There are only finitely many $p$-periodic sequences, so there are $a<$ $b<0$ such that

$$
\tau_{b}=\tau_{a} .
$$

We can even find such $a$ and $b$ that satisfy $a-b \leq-2$. For every $i<0$ and every $j \geq 0$ the local rule gives $\tau_{i}(j+1)=\tau_{i}(j) \oplus \tau_{i+1}(j)$, where $\oplus$ denotes the modulo 2 sum, so that

$$
\tau_{i+1}(j)=\tau_{i}(j) \oplus \tau_{i}(j+1) .
$$

Therefore, for all $a^{\prime}<b^{\prime}<0$, it holds that

$$
\tau_{a^{\prime}}=\tau_{b^{\prime}} \Longrightarrow \tau_{a^{\prime}+1}=\tau_{b^{\prime}+1} .
$$

Using this repeatedly starting on $\tau_{a}$ and $\tau_{b}$ we obtain that $\tau_{a-b}=\tau_{0}$. Because of rule 51 or 255 at cell 0 , the trace $\tau_{a-b}=\tau_{0}$ is either an alternating sequence of 0 's and 1's, or a 


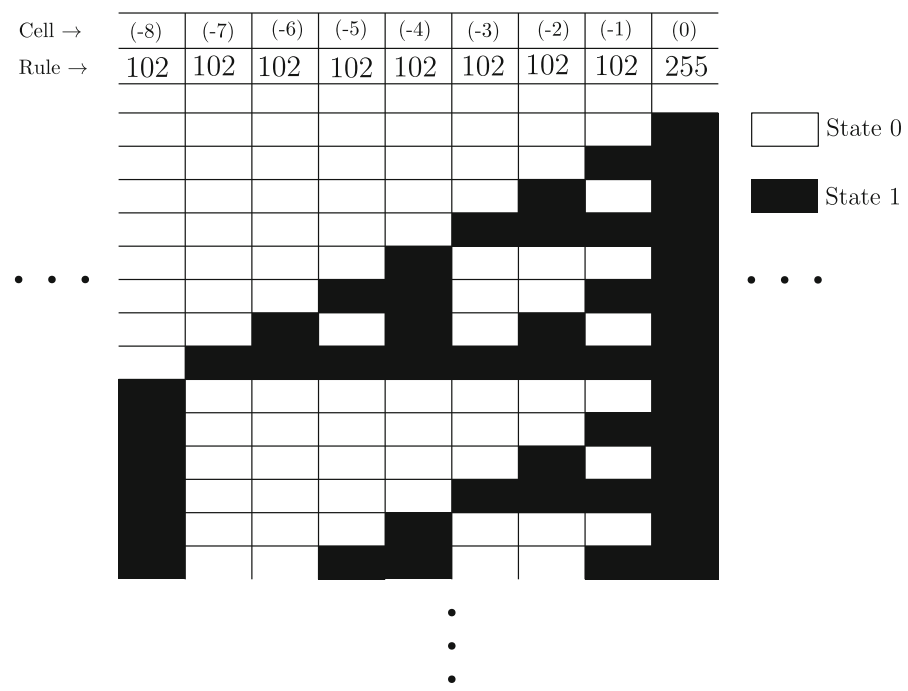

Fig. 5 Snap-shot of a space time diagram of the distribution $(\cdots 102,102,102,255, *, *, \cdots)$

sequence of only 1's. In either case, using (2) we first obtain that $\tau_{a-b+2}$ is a sequence of only 0 's, and then that

$$
(\forall j \in \mathbb{N}) \tau_{0}(j)=0 .
$$

This is contradiction with the fact that the rule 51 or 255 in cell 0 forces states 1 in the trace. Hence, the CA does not have a temporally periodic configuration.

By choosing the local rules of cells $i>0$ in the proposition suitably we can obtain a NUCA with a finite rule distribution, or a bijective and equicontinuous NUCA.

Corollary 1 The NUCA with the 102-finite rule distribution

$$
\text { ...102102102255102102 _.. }
$$

does not have any temporally periodic points. Also, the equicontinuous bijective NUCA with the rule distribution

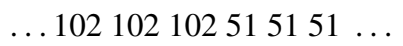

does not have any temporally periodic points.

Note that NUCA ... $102102102515151 \ldots$ is equicontinuous because the trace of cell $i<0$ is uniquely determined by the initial states $c_{i}, \ldots, c_{0}$, and the trace of cell $i \geq$ 0 only depends on $c_{i}$. Recall how this is in contrast to the uniform case where among equicontinuous UCA all configurations are eventually temporally periodic [15]. Note also that NUCA . . 102102102515151 . . is bijective but it is not reversible since the 0 -uniform configuration $\tilde{0}$ and the 1 -uniform configuration $\tilde{1}$ are mapped into configurations that agree with each other at all cells $i<0$. This means that in the inverse function $F_{b}^{-1}$ each cell $i<0$ needs to have in its neighborhood some cell $j \geq 0$, which implies that no finite set of local rules can provide a NUCA for the inverse.

However, it is even possible to find a reversible equicontinuous NUCA that does not have any temporally periodic points: 
Example 1 Let $A=\{0,1\}^{2}$, and consider the NUCA $F$ over $A$ whose cells $i<0$ use radius- $\frac{1}{2}$ local rule $f((a, b),(c, d))=(b, a \oplus d)$ and cells $i \geq 0$ use the radius-0 rule $g(a, b)=(b, a \oplus 1)$. The local rule $f$ of the negative cells is the rule of a one-sided uniform $\mathrm{CA}$ that is reversible and expansive, see [4]. First, $F$ is equicontinuous since the trace of cell $i<0$ is uniquely determined by the initial states $c_{i}, \ldots, c_{0}$, and the trace of cell $i \geq 0$ only depends on $c_{i}$. Second, $F$ is reversible: its inverse is the NUCA that uses in cells $i \geq 0$ the radius- 0 rule $(a, b) \mapsto(b \oplus 1, a)$ and in cells $i<0$ the radius- $\frac{1}{2}$ local rule $((a, b),(c, d)) \mapsto(b \oplus c, a)$. Finally, a similar argument as in Proposition 1 shows that there are no temporally periodic points. The key observation is the fact that, for $i<0$, if $\tau_{i}(j-1)=(a, b), \tau_{i}(j)=(b, c)$, and $\tau_{i}(j)=(c, d)$ are three consecutive elements of the trace at cell $i$ then the element $\tau_{i+1}(j)=(a \oplus c, b \oplus d)$ of the trace of cell $i+1$ is determined. Hence, as in the proof of Proposition 1, among the traces for any temporally periodic initial configuration $c$ there is some $\tau_{k}=\tau_{0}$ for $k \leq-2$. Regardless of the initial configuration, the trace $\tau_{0}$ at cell 0 is the 4 -periodic sequence ..., $(0,0),(0,1),(1,1),(1,0), \ldots$ But then $\tau_{k+1}$ is the $(1,1)$-uniform sequence, and consequently $\tau_{k+2}, \ldots, \tau_{0}$ are $(0,0)$-uniform, which is a contradiction.

With the help of Proposition 1 we can now proceed to prove that it is undecidable if a NUCA with a given finite distribution of rules has any temporally periodic points.

Proposition 2 It is undecidable if the NUCA with the $f$-finite distribution

$$
\ldots f f f g f f f \ldots
$$

for given local rules $f, g$ has any temporally periodic points.

Proof Let $T$ be any given NW-deterministic tile set, let $f_{0}$ and $g_{0}$ be the local rules of ECA 102 and 255, respectively, and let $F_{0}$ be the non-uniform CA with the binary state set $\{0,1\}$ and the distribution $\ldots f_{0} f_{0} f_{0} g_{0} f_{0} f_{0} f_{0} \ldots$ of rules where rule $g_{0}$ is applied at cell 0 . By Proposition 1 the NUCA $F_{0}$ does not have any periodic points. We construct a NUCA $F$ with the state set $A=T \cup\{0,1\}$ where we can assume that $0,1 \notin T$. Let us call the tiles of $T$ as type 1 states and $\{0,1\}$ as type 2 states. The local rule of cell $i$ has radius 1 and it is defined as follows:

- If the neighborhood $i-1, i, i+1$ contains both type 1 and type 2 states, then the next state of cell $i$ is state 0 .

- If all neighbors are of type 1 then, for $a \in T$ and $b \in T$ the current states of the cell and the right neighbor, respectively,

- if there is a matching tile $c$ in Fig. 2 then $c$ is the new state of cell $i$,

- if no matching tile $c$ exists, then the next state is state 0 .

- If all neighbors $i-1, i, i+1$ are of type 2, apply the local rule of $F_{0}$, that is, apply $g_{0}$ if $i=0$ and $f_{0}$ if $i \neq 0$.

We have that rule $g$ of cell $i=0$ is different from the local rule $f$ of the other cells $i \neq 0$. Let us prove that $F$ has a temporally periodic point if and only if $T$ admits a periodic tiling.

If $T$ admits a valid two-way periodic tiling of the plane then the consecutive diagonals of the tiling, as discussed in Sect.2.2, form a periodic orbit of the NUCA, so the diagonals are temporally periodic configurations of the NUCA.

Conversely, suppose there exists a temporally periodic configuration $c \in A^{\mathbb{Z}}$. If $c$ only contains type 2 states then the forward orbit of $c$ is the same as the orbit of $c$ under NUCA $F_{0}$ and, as proved in Proposition 1, configuration $c$ cannot be temporally periodic. If $c$ contains 
both type 1 and type 2 states then there is a cell $i$ such that $c_{i}$ is of type 1 but $c_{i-1}$ or $c_{i+1}$ is of type 2. As type 2 states spread, $F^{n}(c)_{i}$ is of type 2 for all $n \geq 1$, and again $c$ cannot be temporally periodic. We conclude that a temporally periodic $c$ can only contain type 1 states, that is, all states that appear in the orbit of $c$ are tiles from the tile set $T$. The bi-infinite periodic orbit containing $c$ then provides a valid periodic tiling of the plane whose diagonals are the consecutive configurations of the orbit.

Therefore, $T$ admits a periodic tiling if and only if the NUCA has a temporally periodic point. Since the problem of establishing whether a given NW-deterministic tile set admits a periodic tiling is undecidable [17], the claim is proved.

\section{Nilpotency in non-uniform CAs}

In this section we assume a fixed finite set $R$ of local rules over a state set $A$, and we consider the set $\mathcal{N} \subseteq R^{\mathbb{Z}}$ of distributions $b$ such that NUCA $F_{b}$ is nilpotent. Analogous sets of distributions that provide a desired property have been considered in the literature for other properties such as injectivity and surjectivity, and for equicontinuity and sensitivity to initial conditions among linear CA [7]. In these cases the corresponding sets of distributions are recognized by a finite state $\zeta$-automaton, and are hence $\zeta$-rational. (See [18] for details on $\zeta$-rationality.) For surjectivity the corresponding set of distributions is even closed in the usual prodiscrete topology of $A^{\mathbb{Z}}$.

The following simple example shows that $\mathcal{N}$ may be neither open nor closed in the prodiscrete topology.

Example 2 Let $f$ and $g$ be the local rules of ECA 255 and ECA 254, respectively, so that $f(a, b, c)=1$ for all $a, b, c \in\{0,1\}$, and $g(a, b, c)=0$ only for $a=b=c=0$. Every $f$-finite distribution defines a nilpotent NUCA since in one step rule $f$ creates states 1 at all but a finite number of cells, and then in finite time these states 1 spread to all cells. On the other hand, all $g$-finite distributions define non-nilpotent NUCAs since the 0 -uniform initial configuration never reaches a fixed point. Since $f$-finite and $g$-finite configurations are both dense in $\{f, g\}^{\mathbb{Z}}$, we see that $\mathcal{N}$ is neither open nor closed in the prodiscrete topology.

We start by proving that if all periodic distributions over $R$ are in $\mathcal{N}$, so are all eventually periodic distributions.

Proposition 3 If $F_{a}$ is nilpotent for every periodic distribution $a \in R^{\mathbb{Z}}$ then $F_{b}$ is nilpotent for every eventually periodic distribution $b \in R^{\mathbb{Z}}$.

Proof Let $R$ contain local rules of radius $r$. Assume that $F_{a}$ is nilpotent for all periodic $a$, and let $b=\ldots p$ p uq $q \ldots$ be an arbitrary eventually periodic distribution, $p, q \in R^{+}$and $u \in R^{*}$. Let $\tilde{p}$ and $\tilde{q}$ be the periodic distributions $\tilde{p}=\ldots p p p \ldots$ and $\tilde{q}=\ldots q q q \ldots$, where we align $p$ 's and $q$ 's in the same positions as in the tails of $b$ on the left and on the right, respectively.

Due to nilpotency of $F_{\tilde{p}}$ and $F_{\tilde{q}}$ there exist configurations $c_{p}$ and $c_{q}$ and a constant $n$ such that $F_{\tilde{p}}^{n}(c)=c_{p}$ and $F_{\tilde{q}}^{n}(c)=c_{q}$ for all initial configurations $c$. There is then number $M>0$ such that for all $c$ it holds that

$$
F_{b}^{n}(c)(i)= \begin{cases}c_{p}(i) & \text { for } i<-M \\ c_{q}(i) & \text { for } i>M .\end{cases}
$$


Note that (3) holds for all configurations $c$, so by applying (3) to initial configurations $F_{b}(c), F_{b}^{2}(c), \ldots$ in place of $c$ we see that (3) also holds for any $t \geq n$ in place of $n$ :

$$
(\forall t \geq n) F_{b}^{t}(c)(i)= \begin{cases}c_{p}(i) & \text { for } i<-M, \\ c_{q}(i) & \text { for } i>M .\end{cases}
$$

Local rules in $R$ have radius $r$, so changing the distribution $b$ at cells $>M+r+n r$ will not influence by time $n$ the states of cells $\leq M+r$. Analogous fact holds on the negative side. Hence, for any distribution $b^{\prime}$ that agrees with $b$ in cells $-(M+r+n r), \ldots, M+r+n r$, we have for any configuration $c$ that

$$
F_{b^{\prime}}^{n}(c)(i)=F_{b}^{n}(c)(i) \text { for }-(M+r) \leq i \leq M+r .
$$

By (3) and (5) we have that $F_{b^{\prime}}^{n}(c)(i)=c_{p}(i)$ when $-(M+r) \leq i<-M$ and $F_{b^{\prime}}^{n}(c)(i)=$ $c_{q}(i)$ when $M<i \leq M+r$. This is true for all configurations $c$, so again by applying it to configurations $F_{b}(c), F_{b}^{2}(c), \ldots$ in place of $c$, we have that for all $t \geq n$ holds

$$
F_{b^{\prime}}^{t}(c)(i)=F_{b}^{t}(c)(i)=\left\{\begin{array}{llr}
c_{p}(i) & \text { for } & -(M+r) \leq i<-M, \\
c_{q}(i) & \text { for } & M<i \leq M+r .
\end{array}\right.
$$

Since $r$ is the radius of the local rules we can infer from (5) and (6), inductively for $t=$ $n+1, n+2, \ldots$, that for all $t \geq n$

$$
F_{b^{\prime}}^{t}(c)(i)=F_{b}^{t}(c)(i) \text { for }-(M+r) \leq i \leq M+r .
$$

Equality (7) holds for any distribution $b^{\prime}$ that agrees with $b$ in cells $-(M+r+n r), \ldots, M+$ $r+n r$. Among such distributions there clearly is a periodic $b^{\prime}$ with repeating period $p \ldots$ p $и q q \ldots q$ (with sufficiently many $p$ 's and $q$ 's). Since NUCA with periodic distribution $b^{\prime}$ is nilpotent, there is a configuration $c^{\prime}$ and constant $m \geq n$ such that $F_{b^{\prime}}^{m}(c)=c^{\prime}$ for all $c \in A^{\mathbb{Z}}$. Putting this together with (7) gives that $F_{b}^{m}(c)(i)=c^{\prime}(i)$ for $-(M+r) \leq i \leq M+r$, and finally with (4) we have that $F_{b}^{m}(c)$ is independent of $c$, that is, $F_{b}$ is nilpotent.

Note that Theorem 6.1 in [6] implies the analogous result for equicontinuity: if $F_{a}$ is equicontinuous for every periodic distribution $a \in R^{\mathbb{Z}}$ then $F_{b}$ is equicontinuous for every eventually periodic distribution $b \in R^{\mathbb{Z}}$.

One may wonder whether nilpotency of all periodic distributions implies that all distributions are nilpotent. In the following we construct an example showing that this is not necessarily the case. In the construction we rely on the structure of valid tilings by Robinson's aperiodic Wang tile set [19], and the NW-deterministic variant of this set [13].

We first review some relevant features of Robinson's tiles, using the terminology of [19]. The tile set consists of crosses (Fig. 6) and arms (Fig. 7). The tiles may be rotated, so each of the six arms in Fig. 7 come in four different orientations. The matching rule is that the arrows must continue across tile boundaries so that arrow heads meet arrow tails. The arrows on the horizontal and vertical center lines of the tiles are central arrows, and the arrows on the sides are side arrows. A cross faces the two directions of its side arrows. There are some additional layers of arrows on the tiles that we do not draw: a parity layer that guarantees a regular lattice of crosses on every second row and column, and diagonal signals introduced in [13] to make the tile set NW-deterministic.

For each $n \geq 1$, special squares of size $\left(2^{n}-1\right) \times\left(2^{n}-1\right)$ can be constructed recursively. Such a square is called a level $n$ square. Level 1 squares are crosses. At level $n+1$, four level $n$ squares are placed facing each other and with a cross in the middle (Fig. 8). The central cross can be oriented in any direction. 


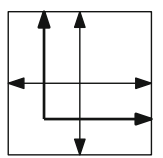

(a)

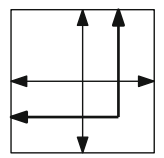

(b)

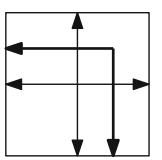

(c)

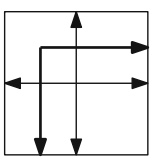

(d)

Fig. 6 Crosses, in all four different orientations
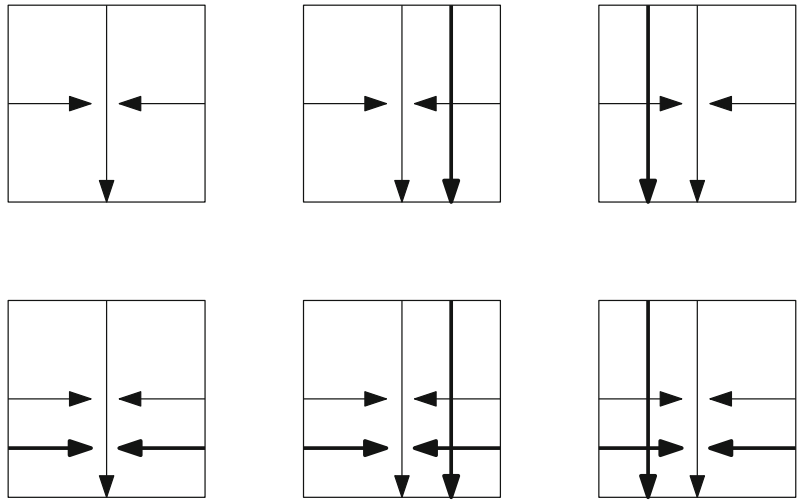

Fig. 7 Arms. Each arm can be rotated in four orientations

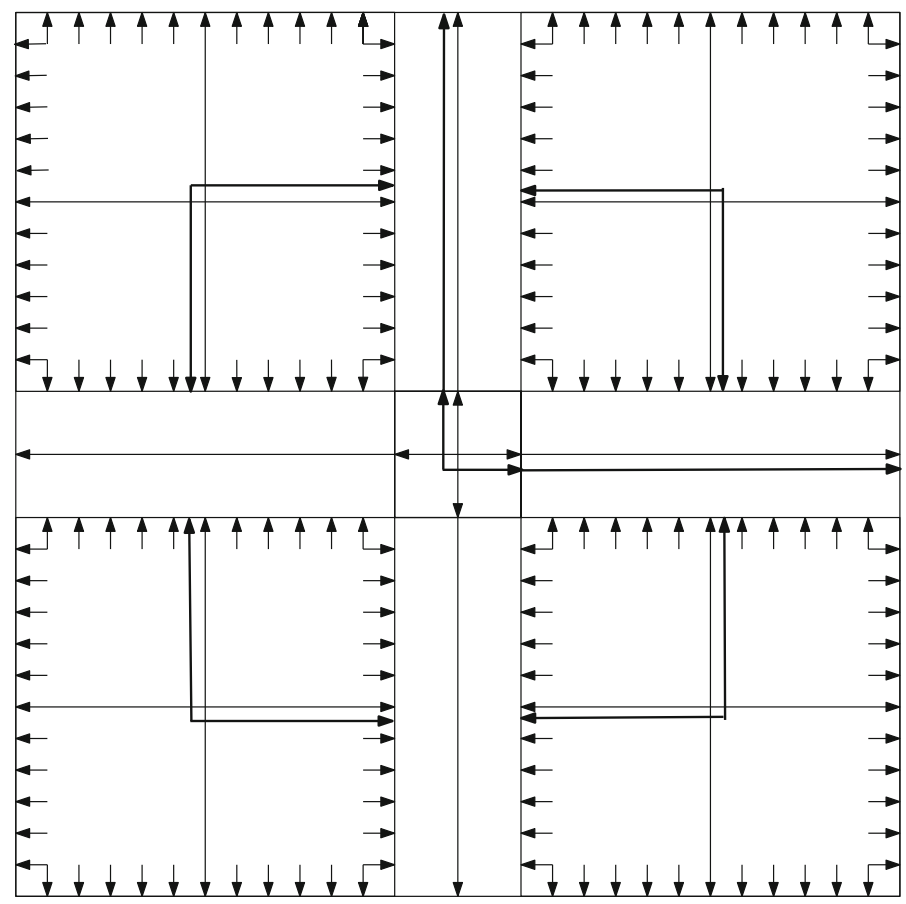

Fig. 8 Recursive construction of level $n+1$ square from four level $n$ squares facing each other along with a cross in the middle 
Fig. 9 Nested borders formed by the side arrows

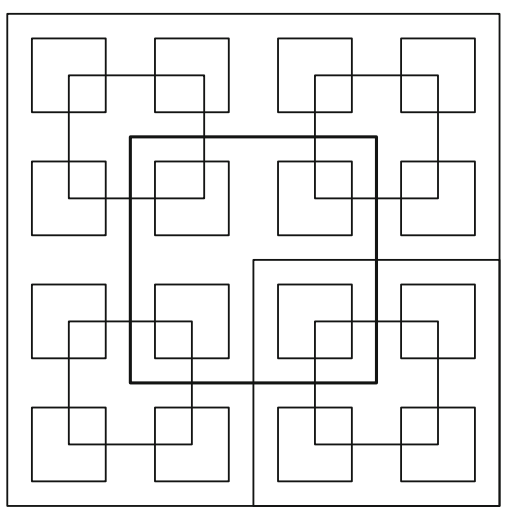

The special squares can be constructed at all levels without tiling errors. In the limit, one obtains a valid tiling of the plane. Moreover, it is proved in [19] that every valid tiling of the plane must contain special squares of all levels, proving that no tiling can be periodic (the center lines of special squares only contain one cross so there can be no horizontal or vertical period in a valid tiling). This implies that the Robinson's tile set is aperiodic.

The side arrows draw in special squares a hierarchy of square shaped borders, see Fig. 9. The north (south) segments of these borders consist of precisely those tiles that have a horizontal side arrow above (below, respectively) the center line of the tile.

Lemma 1 Let $T$ be the (NW-deterministic) Robinson's tile set, and let A and B be the disjoint subsets of $T$ containing those tiles that have a horizontal side arrow above or below the center line, respectively.

(a) There is a valid tiling by $T$ such that no horizontal row contains tiles from both $A$ and $B$.

(b) For every valid tiling and every even integer $m>0$, there exists $i \in \mathbb{Z}$ such that row $i$ contains a tile from $B$ and row $i+m$ contains a tile from $A$.

(c) For every even integer $m>0$ there exists integer $M$ such that every correctly tiled $M \times M$ square has some rows $i$ and $i+m$ containing a tile from $B$ and a tile from $A$, respectively.

Proof In the special squares the nested square borders are aligned so that no horizontal row contains both a top segment of a border ( $A$ tiles) and a bottom segment of a border ( $B$ tiles). In the limit one obtains a valid tiling where (a) holds.

To prove (b), write $m=k 2^{n}$ for $k$ odd and $n \geq 1$. In the special square of level $n+1$ there are horizontal rows $j$ and $j+2^{n}$ containing a tile from $B$ and $A$, respectively. (These are the two rows containing the centers of the four level $n$ squares facing each other, see Fig. 8.) For every $t \geq 0$, the level $n+t+1$ special square contains $2^{t}$ level $n+1$ squares on top of each other. The rows $j$ and $j+2^{n}$ of these squares form a sequence of rows $j, j+2^{n}, j+2 \cdot 2^{n}, \ldots, j+\left(2^{t+1}-1\right) \cdot 2^{n}$ that alternatingly contain a tile from $B$ and a tile from $A$. Choosing $t$ such that $2^{t+1}-1 \geq k$ we have row $j$ containing a tile from $B$ and row $j+k 2^{n}=j+m$ containing a tile of $A$. Since every valid tiling contains special squares of all levels, claim (b) follows.

Claim (c) now follows from (b) using compactness: suppose (c) is false so that there is an even $m>0$ such that for every $M$ there is a correctly tiled $M \times M$ square $s_{M}$ without any rows at distance $m$ containing a $B$ and an $A$ tile. Centering these squares at the origin, letting $M$ grow and taking the limit of a converging subsequence we obtain a valid tiling $t$ of the plane whose every finite pattern appears in some square $s_{M}$. By (b) there are some $x, y, i \in \mathbb{Z}$ 
such that $t(x, i) \in B$ and $t(y, i+m) \in A$. But a finite pattern of $t$ containing cells $(x, i)$ and $(y, i+m)$ appears in some square $s_{M}$, contradicting the choice of these squares.

Now we are ready to construct NUCAs from the NW-deterministic Robinson tile set.

Proposition 4 There are two local rules $f$ and $g$ such that $F_{b}$ is nilpotent for every periodic distribution $b \in\{f, g\}^{\mathbb{Z}}$ but $F_{b}$ is not nilpotent, or even equicontinuous, for some (nonperiodic) distribution $b \in\{f, g\}^{\mathbb{Z}}$.

Proof Let $T$ be the NW-deterministic Robinson's tile set, and let $A$ and $B$ be the disjoint subsets of $T$ containing those tiles that have a horizontal side arrow above or below the center line, respectively. The state set of our NUCAs is $S=T \cup\{q\}$ where $q \notin T$. Local rules $f$ and $g$ use radius- $\frac{1}{2}$ neighborhood. The rules are identical to the rule $f_{T}$ given by (1) in Sect. 2.2 with the following exceptions:

$$
\begin{aligned}
& (\forall a \in A)(\forall s \in S) f(a, s)=q, \\
& (\forall b \in B)(\forall s \in S) g(b, s)=q .
\end{aligned}
$$

Let us first prove that there is a rule distribution $b \in\{f, g\}^{\mathbb{Z}}$ such that $F_{b}$ is not nilpotent, or even equicontinuous. By Lemma 1(a) there is a valid tiling $t$ where no row contains both a tile of $A$ and a tile of $B$. Let us choose a rule distribution $b$ such that if row $i$ contains a tile of $A$ then $b_{i}=g$ and if row $i$ contains a tile of $B$ then $b_{i}=f$. For rows $i$ without tiles of $A$ or $B$ the rule $b_{i}$ can be chosen arbitrarily.

Tiling $t$ yields then a valid space-time diagram of NUCA $F_{b}$ where consecutive diagonals are the consecutive configurations in the orbit: for any $n \in \mathbb{Z}$ the configuration $c^{(n)}$ at time $n$ is the $n$ 'th diagonal, i.e., $c_{i}^{(n)}=t(n+i, i)$ for all $i \in \mathbb{Z}$. Note that the states that any cell $i$ obtains in configurations $c^{(n)}$ are the tiles of the $i$ 'th row of tiling $t$. This means that a cell with local rule $f$ never has state from set $A$ and a cell with local rule $g$ never has state from set $B$, so that the special rules (8) are not applicable in any cell in any configuration $c^{(n)}$. Consequently, each cell applies the local rule $f_{T}$ given by (1) which means that $F_{b}\left(c^{(n)}\right)=c^{(n+1)}$ for all $n \in \mathbb{Z}$. Because the NUCA $F_{b}$ has a fixed point configuration . .qqq $\ldots$ that this orbit never reaches, the NUCA is not nilpotent. It is not even equicontinuous since the state $q$ spreads to the left, so that changing in configuration $c^{(0)}$ the state of any cell $i>0$ to $q$ causes a change in the trace $\tau_{0}$ of cell 0 in the orbit of $c^{(0)}$.

Let us prove next that all periodic distributions yield a nilpotent NUCA. Let $b \in\{f, g\}^{\mathbb{Z}}$ be periodic so that for some even number $m>0$ we have $\sigma^{m}(b)=b$. Let $M$ be the number provided by Lemma 1(c) for this number $m$.

We claim that in NUCA $F_{b}$ every initial configuration becomes the $q$-uniform configuration $\tilde{q}$ within $2 M$ steps. Suppose the contrary: for some $c \in S^{\mathbb{Z}}$ and cell $k \in \mathbb{Z}$ we have that $F_{b}^{2 M}(c)(k) \neq q$. Let us denote $c^{(n)}=F_{b}^{n}(c)$ for $n \geq 0$, and let $t \in S^{\mathbb{Z}^{2}}$ be a two-dimensional configuration whose consecutive diagonals contain consecutive configurations of the forward orbit of $c$. More precisely, $t(n+i, i)=c_{i}^{(n)}$ for all $n \geq 0$ and $i \in \mathbb{Z}$, and the values $t(n+i, i)$ for $n<0$ may be chosen arbitrarily.

We have that $t(2 M+k, k) \neq q$. Because states $q$ spread with speed one to the left, we then also have that

$$
t(x, y) \neq q \text { when } M+k \leq x \leq 2 M+k \text { and } k \leq y \leq k+M .
$$

This means that $t(x, y)$ for $M+k \leq x<2 M+k$ and $k \leq y<k+M$ form an $M \times M$ square that is correctly tiled by $T$. By Lemma 1(c) there are two rows in this square at distance $m$ that contain an element of $A$ and an element of $B$, respectively. Since distribution $b$ has period 
$m$, both rows correspond to cells that apply the same local rule, $f$ or $g$. In any case, one of these cells enters state $q$ by the special update rule (8). This means that $t(x, y)=q$ for some $x, y$ in the intervals $M+k \leq x \leq 2 M+k$ and $k \leq y \leq k+M$, which contradicts (9). We conclude that NUCA $F_{b}$ is nilpotent.

\section{Conclusions}

The paper deals with temporal periodicity and nilpotency in non-uniform one-dimensional CA. Some significant differences can be seen between non-uniform and uniform CA. In contrast to the classical uniform case, there are NUCAs- even reversible equicontinuous ones-that do not have any temporally periodic points. Moreover, whether a given NUCA has any temporally periodic points turns out to be undecidable. The undecidability holds for NUCAs with very simple rule distributions so it is not due to non-recursiveness hidden in the distribution. Whether undecidability holds also for bijective or reversible NUCAs remains for future study.

We also considered the effect of local rule distributions on nilpotency of NUCAs. Using Robinson's aperiodic tile set we showed that there is a pair of local rules such that some distribution of the rules defines a non-nilpotent NUCA even though every periodic distribution leads to a nilpotent NUCA. We proved, however, for any collection of local rules that nilpotency on all periodic distributions implies nilpotency on all eventually periodic distributions.

Funding Open access funding provided by University of Turku (UTU) including Turku University Central Hospital.

Open Access This article is licensed under a Creative Commons Attribution 4.0 International License, which permits use, sharing, adaptation, distribution and reproduction in any medium or format, as long as you give appropriate credit to the original author(s) and the source, provide a link to the Creative Commons licence, and indicate if changes were made. The images or other third party material in this article are included in the article's Creative Commons licence, unless indicated otherwise in a credit line to the material. If material is not included in the article's Creative Commons licence and your intended use is not permitted by statutory regulation or exceeds the permitted use, you will need to obtain permission directly from the copyright holder. To view a copy of this licence, visit http://creativecommons.org/licenses/by/4.0/.

\section{References}

1. Aanderaa, S., Lewis, H.: Linear sampling and the $\forall \exists \forall$ case of the decision problem. J. Symb. Log. 39(3), 519-548 (1974)

2. Berger, R.: The Undecidability of the Domino Problem. Memoirs of the American Mathematical Society. American Mathematical Society, Providence (1966)

3. Boyle, M.: Open problems in symbolic dynamics. In: Brin, M., Burns, K., Dolgopyat, D., Pesin, Y. (eds.) Geometric and Probabilistic Structures in Dynamics, Contemporary Mathematics, vol. 469, pp. 69-118. American Mathematical Society, Providence (2008)

4. Boyle, M., Maass, A.: Expansive invertible onesided cellular automata. J. Math. Soc. Jpn. 52(4), 725-740 (2000)

5. Ceccherini-Silberstein, T., Coornaert, M.: Cellular Automata and Groups. Springer Monographs in Mathematics. Springer, Berlin (2010)

6. Dennunzio, A., Formenti, E., Provillard, J.: Non-uniform cellular automata: classes, dynamics, and decidability. Inf. Comput. 215, 32-46 (2012)

7. Dennunzio, A., Formenti, E., Provillard, J.: Local rule distributions, language complexity and non-uniform cellular automata. Theor. Comput. Sci. 504, 38-51 (2013) 
8. Dennunzio, A., Formenti, E., Provillard, J.: Three research directions in non-uniform cellular automata. Theor. Comput. Sci. 559, 73-90 (2014)

9. Devaney, R.: An Introduction to Chaotic Dynamical Systems. Addison-Wesley, Boston (1989)

10. Durand, B., Formenti, E., Varouchas, G.: On undecidability of equicontinuity classification for cellular automata. In: Morvan, M., Rémila, E. (eds.) Discrete Models for Complex Systems, DMCS'03, DMTCS Proceedings, vol. AB, pp. 117-128. DMTCS (2003)

11. Guillon, P., Richard, G.: Nilpotency and limit sets of cellular automata. In: Ochmański, E., Tyszkiewicz J. (eds.) Mathematical Foundations of Computer Science 2008, MFCS 2008, Lecture Notes in Computer Science, vol. 5162, pp. 375-386. Springer, Berlin (2008)

12. Jeandel, E., Rao, M.: An aperiodic set of 11 Wang tiles (2015). arXiv preprint arXiv: 1506.06492

13. Kari, J.: The nilpotency problem of one-dimensional cellular automata. SIAM J. Comput. 21(3), 571-586 (1992)

14. Kari, J.: Rice's theorem for the limit sets of cellular automata. Theor. Comput. Sci. 127(2), 229-254 (1994)

15. Kůrka, P.: Languages, equicontinuity and attractors in cellular automata. Ergod. Theory Dyn. Syst. 17(2), 417-433 (1997)

16. Kůrka, P.: Topological and Symbolic Dynamics. Collection SMF, Société mathématique de France (2003)

17. Mazoyer, J., Rapaport, I.: Global fixed point attractors of circular cellular automata and periodic tilings of the plane: undecidability results. Discret. Math. 199(1-3), 103-122 (1999)

18. Perrin, D., Pin, J.É.: Infinite Words: Automata, Semigroups, Logic and Games. Pure and Applied Mathematics Series, vol. 141. Elsevier Morgan Kaufmann, Burlington (2004)

19. Robinson, R.M.: Undecidability and nonperiodicity for tilings of the plane. Invent. Math. 12(3), 177-209 (1971)

20. Wolfram, S.: Statistical mechanics of cellular automata. Rev. Mod. Phys. 55, 601-644 (1983)

Publisher's Note Springer Nature remains neutral with regard to jurisdictional claims in published maps and institutional affiliations. 